\title{
The Embodiment Design of a Two-Wheeled Self-Balancing Robot
}

\author{
Alessio Salerno \\ Department of Mechanical Engineering \& \\ Centre for Intelligent Machines \\ McGill University \\ 817 Sherbrooke West \\ Montreal, QC, H3A 2K6 Canada \\ salernodcim.mcgill.ca
}

(corresponding author)

\author{
Rafael Perlin \\ Ardi Consultants Enr. \\ 14-2560 Mayfair Ave. \\ Montreal, QC, H4B 2C8 Canada \\ rafaelperlin@hotmail.com \\ Jorge Angeles \\ Department of Mechanical Engineering \& \\ Centre for Intelligent Machines \\ McGill University \\ 817 Sherbrooke West \\ Montreal, QC, H3A 2K6 Canada \\ angelesdcim.mcgill.ca
}

\begin{abstract}
The embodiment design of a two-wheeled selfbalancing human augmentation robot for the mobility-challenged is reported. The prototype relies on a dead-reckoning multisensor system consisting of $i$ ) two optical incremental encoders and $i$ ) a solid-state tilt sensor. The command inputs are provided to the robot controller, which is based on PC/104 technology, by means of a RF control unit. After describing the research motivation and application of the system, a set of robot design solutions is outlined along with technical discussions on component layout, payload holder and chassis design issues. A few simulation results on the motion control performance are included as well
\end{abstract}

\section{INTRODUCTION}

This work focuses on the embodiment design of twowheeled mobile robots. In particular we built three different virtual prototypes of the robot at hand and critically analyze them against the desired specifications.

The subject of the paper is Quasimoro, a two-wheeled selfbalancing mobile robot as above. The prototype, currently under development at McGill's Robotic Mechanical Systems Laboratory, will rely on a dead-reckoning multi-sensor system, which will consist of $i$ ) two optical incremental encoders mounted on the motor shafts, to sense the relative angular positions and angular velocities of the wheels with respect to the intermediate body; and $i$ i) a solid-state tilt sensor to measure the inclination and angular velocity (by numerical differentiation) of the intermediate body with respect to the vertical. The absolute angular position (velocity) of the wheels is readily measured as the algebraic sum of the relative wheel positions (velocities) with respect to the intermediate body and the position of the intermediate body itself. The system outputs, i.e. the driving and the steering robot velocity, are provided to the robot controller, which is based on $\mathrm{PC} / 104$ technology, by means of a 2-channel dual-joystick remote control unit.

After describing the research motivation and application of the system, a set of design solutions for the system at hand is outlined. The actuation sub-system of the first design solution consists of a DC brushed motor and its planetary gearhead, which is directly coupled to the wheel hub of a custom-made wheel based on O-ring technology. In order to lower the overall mass centre of the robot, to limit the width of the robot and to contain the bending moment of the planetary gearhead output shaft, a timing belt transmission was used. Moreover, in order to reduce the manufacturing costs a commercial bicycle wheel was chosen, as opposed to the foregoing custom-made one. Hence, the second design solution is characterized by a bicycle wheel tightened to a custom-made shaft by means of a frictional connector; a timing belt takes care of the mechanical power transmission from the output shaft of the planetary gear to the custom-made shaft. Such a solution allows for redundant sensing since motion sensors can be readily assembled on the custom-made shaft. However, another design solution was preferred to the latter. Instead of the custom-made shaft solution, which is rather expensive, a simpler and more cost-effective one was adopted, which uses the bicycle wheel shaft and bearings. This work includes also technical discussions on component layout, payload holder and chassis design issues.

Throughout the work we tried to stick to the "keep it simple stupid!"(KISS) design approach. Most of the robot parts were dimensioned according to standard stress analysis techniques [1].

\section{Motivation AND ApPliCATIONS}

The motivation behind the Quasimoro project is twofold. First we intend to produce an experimental test-bed for

- validation of the advantages introduced by quasiholonomy in the computed torque control of nonholonomic mechanical systems [2];

- analysis of the effect of caster wheels on trajectory tracking of mobile robots;

- control of mobile robots based on the inclusion of the robot dynamics;

- validation of multivariable control strategies for the robot at hand, which feature robustness with respect to payload variation [3]; and

- educational robotics purposes: students can relate to a real robot much better than to a piece of software, a 
working robot program will be much more than just a logic solution coded in software [4].

The second main motivation lies in the application of the robot. Quasimoro is meant to be used as an assistive device for mobility challenged. The final application of the robot will be in a living quarter specifically designed to study how to improve the living conditions of a paraplegic wheelchair user [5].

\section{FIRST DESIGN SOLUTION}

Quasimoro comprises two driving wheels and an intermediate body carrying the payload. As depicted in Fig. 1, the first virtual prototype that we came up with relies on a dead-reckoning multi-sensor system, which will consist of i) two incremental encoders mounted on the motor shafts, to sense the relative angular positions and angular velocities of the wheels with respect to the intermediate body; and ii) a tilt sensor, namely a CXTA01 by Crossbow [6], to measure the inclination and the angular velocity (by numerical differentiation) of the intermediate body (IB) with respect to the vertical. The absolute angular position (velocity) of the wheels is readily measured as the algebraic sum of the relative wheel positions (velocities) with respect to the intermediate body and the position of the intermediate body itself. The system outputs, i.e. the driving and the steering robot velocity, are provided to the robot controller, a PC/104, by means a remote control unit.

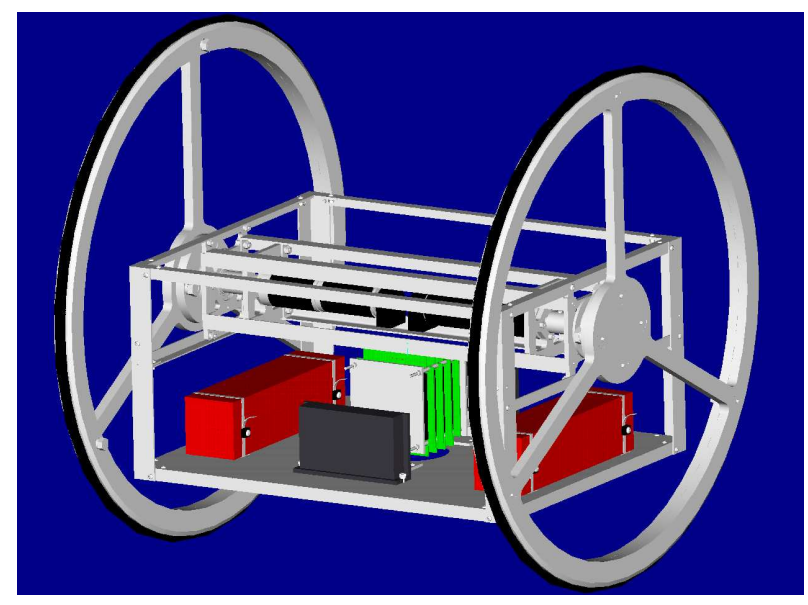

Fig. 1. Robot digital mock-up — first design solution

\section{A. Wheel Design}

The first wheel design that we came up with consisted of two custom-made components (18 and 19 of Fig. 2), an elastomeric O-ring (15 of Fig, 2) and three nut-bolt sets (7 and 13 of Fig, 2). The O-ring plays the role of the wheel tire. The toroidal shape of the O-ring strongly reduces the rolling friction force between wheel and ground, thus increasing the accuracy of robot positioning. The three nut-bolt sets were designed to ease the O-ring servicing.

One of the main challenges faced here was the O-ring dimensioning, which was carried out at a major pioneering

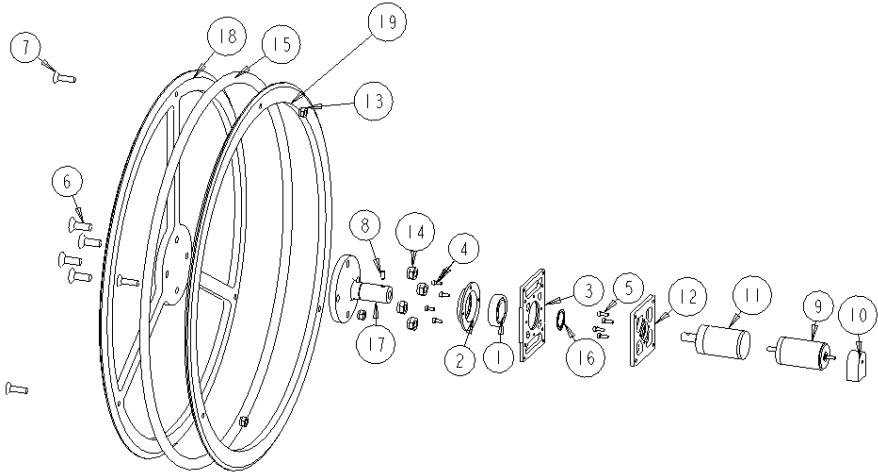

Fig. 2. Wheel sub-assembly exploded view — first design solution

level [7]. In fact, elastomeric O-rings are meant to be used in sealing applications, cushion installations, and low-power drive belt transmissions. While a selection procedure of $\mathrm{O}$ rings used for standard applications is readily available [7], no methodology can be found in the literature for selecting O-rings to be used in wheel-tire applications.

1) O-Ring Dimensioning: In selecting the O-ring we followed a procedure stemming from the integration of standard design methodologies for some mechanical systems, such as rotary seals [7], drive belts [8] and band brakes [1], whose dynamics resemble the one of our system.

The first step consists in determining the O-ring compound, as selected compound may have significant influence on groove design. Polyurethane was chosen for its excellent wear resistance, high tensile strength and high elasticity in comparison with any other elastomer [7]. Moreover, the heat resistance $\left(82^{\circ} \mathrm{C}\right)$ and cold flexibility $\left(-40^{\circ} \mathrm{C}\right)$ are also high enough to safely use polyurethane in the environment in which the robot navigates. Polyester urethane is preferred to polyether urethane for its better resistance to water; in fact, wet floors and humid areas sometimes present in a living quarter might be harmful for the robot tires. Furthermore, polyurethane features also a chemical resistance to ozone and aging and excellent dynamic properties [7]. Such a compound selection complies also with the general requirements for elastomer drive belt materials [7].

For the given application of the robot and the corresponding design specifications on $a$ ) ground clearance, $b$ ) position of the overall mass centre of the robot under full payload and c) door negotiation, the wheel diameter should be at least $590 \mathrm{~mm}$ [5], which represents the minimum desired dimension of the outer diameter (OD) of the O-ring, as installed in the groove. Off-the-shelf O-rings for sealing applications are discarded because of their small cross section (CS) diameter; in fact, the biggest CS diameter available is only $6.985 \mathrm{~mm}$, which does not guarantee a sufficient resistance to compression. Custom-made O-rings are discarded as well, since they are too expensive. Therefore, we selected O-rings for drivebelt applications; in particular we selected a 95 Shore A durometer (the highest hardness available) O-ring of $3 / 4^{\prime \prime}$ of CS diameter (the biggest available) and $11.22^{\prime \prime}$ of length by Tampa Rubber [9]. 
2) Groove Dimensioning: After having selected the O-ring, its groove needs to be designed. The latter was designed under the guidelines below:

- under minimum payload, there should be at least $0.2 \mathrm{~mm}$ of radial compression of the tire cross section; by doing so the O-ring will not quickly take a $100 \%$ compression $\operatorname{set}^{1}[7]$;

- the groove should accommodate an internal diameter (ID) of the O-ring (as installed in the groove) of $5 \%$. This percentage was chosen so as $i$ ) to have the running and break-out friction O-ring/groove as high as possible and ii) not to shorten the life of the O-ring. The percent reduction in cross section diameter of the O-ring corresponding to the $5 \%$ ID stretch, turns out to be $2.41 \%$, computed from the loss of compression diameter due to stretch formula [7];

- to guarantee that the ID expansion needed to reach the groove during assembly does not exceed $50 \%$ of the ultimate elongation of the polyurethane [7].

\section{B. Actuation System Dimensioning}

The Quasimoro actuation system consists of two identical units; each of them endowed with $i$ ) a Maxon RE 40 (148867) brushed motor (9 in Fig. 2), equipped with a planetary gearhead (PG), namely, a Maxon GP 42 C (203123) (11 in Fig. 2); and $i$ ) a digital encoder HP HEDS N5540 A11 (10 in Fig. 2). The PG is secured to the motor mount (12 of Fig. 2) by means of four screws ( 5 of Fig. 2). A rolling bearing (1 in Fig. 2) and a custom-made wheel hub (11 in Fig. 2) were added in order to reduce the bending moment of the planetary gearhead output shaft of $30 \%$, approximately. The bearing is mounted on a custom-made housing ( 2 of Fig. 2), which is fastened to a custom-made plate ( 3 of Fig. 2) by means of four screws (4 of Fig. 2). A snap ring (16 of Fig. 2) prevents the bearing from axial motion along the wheel-hub. The driving torque is transmitted to the wheel by means of four nut-bolt sets (6 and 14 of Fig. 2), while the wheel-hub is connected to the PG by menas of a set screw (8 of Fig. 2). A snapshot of the overall motor mount assembly is given in Fig. 3 .

In order to guarantee the wheel rolling under any payload condition, the torque applied to the wheel cannot be higher than $10 \mathrm{Nm}$, value obtained considering the sliding friction coefficient of rubber on rubber [10] and using a safety factor of 1.25. In order to dimension the actuation system we conducted a series of numerical simulations in Matlab 6.5.1.199709 and Simulink 5.1 (R13SP1) using the variable-step solver ODE45 (Dormand-Prince). In order to set-up the simulations we assume that: $i$ ) the robot undergoes motion on a planar surface, which we call $\mathcal{B} ; i i$ ) the robot wheels are assumed to be always in contact with $\mathcal{B}$; and iii) the model does not account for internal dissipation, such as friction, in the bearings. A sketch of the robot is shown in Fig. 4. We define $\mathcal{A}$ and $\mathcal{A}^{\prime}$ as the axis passing through the centers of the wheels

\footnotetext{
${ }^{1}$ The compression set is the amout by which a rubber specimen fails to return to original shape after release of compressive load [7]
}

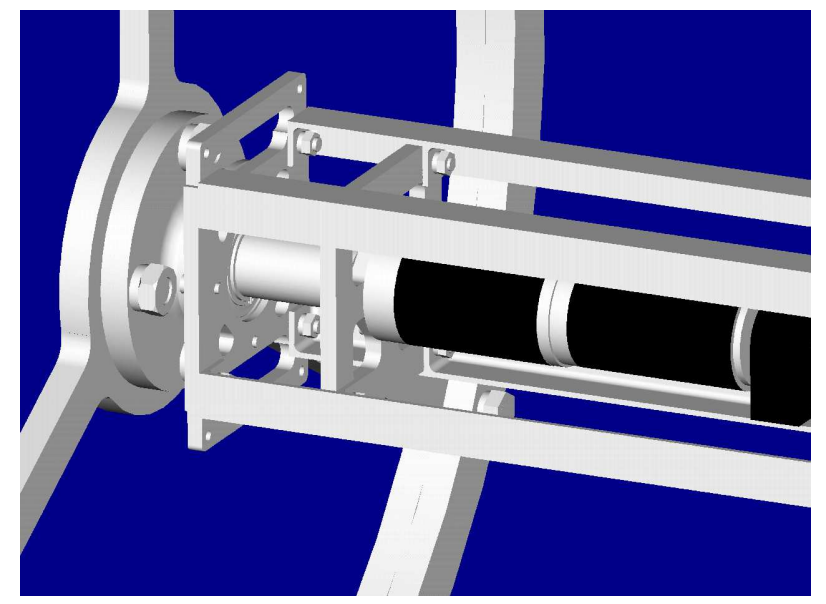

Fig. 3. Motor mount close-up — first design solution

and that parallel to the latter and passing through the center of mass $C_{3}$ of the augmented mass of the IB (i.e. taking into account also the payload), respectively. The chassis of the IB is represented by a cylinder with axis of symmetry $\mathcal{D}$, which is normal to $\mathcal{A}$. We define three orthonormal triads of vectors:

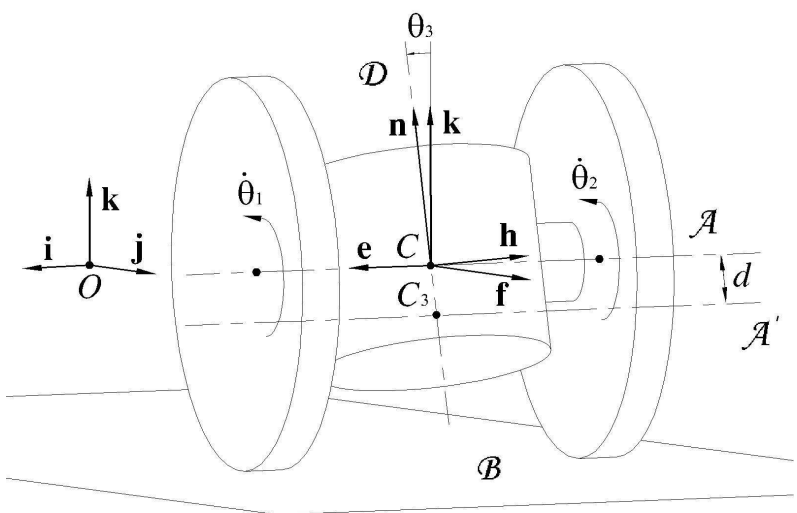

Fig. 4. Sketch of Quasimoro

$\{\mathbf{i}, \mathbf{j}, \mathbf{k}\},\{\mathbf{e}, \mathbf{f}, \mathbf{k}\}$ and $\{\mathbf{e}, \mathbf{h}, \mathbf{n}\}$. The triad $\{\mathbf{i}, \mathbf{j}, \mathbf{k}\}$ defines an inertial frame attached to the ground with origin $O$ and with k vertical, such that points $O$ and $C$ lie at the same level. The frame defined by $\{\mathbf{e}, \mathbf{f}, \mathbf{k}\}$ has its origin at the midpoint $C$ of the line linking the centers of the two wheels; in particular, $\mathbf{e}$ is parallel to $\mathcal{A}$. The frame defined by $\{\mathbf{e}, \mathbf{h}, \mathbf{n}\}$ is attached to the IB and centered at point $C$, while $\mathbf{n}$ lies on the $\mathcal{D}$ axis. We indicate with $\theta_{1}$ and $\theta_{2}$ the angular displacements of the two wheels, while $\mathbf{c}$ is the position vector of point $C$. We define $\theta_{3}$ as the angle of rotation of the IB, also called the tilt angle, about $\mathcal{A}$. We define $J_{1}$ as the moment of inertia of the IB about its axis of symmetry, $J_{2}$ as the moment of inertia of the IB about $\mathcal{A}, d$ is the distance between $C$ and $C_{3}, m_{3}$ is the augmented mass of the IB, $\psi$ as the orientation angle, i.e., the angle between vectors $\mathbf{i}$ and $\mathbf{e}, l$ as the distance between the centers of the wheels, $r$ as the wheel radius, $\rho$ as $r / l$, and assume that the wheels roll without slipping on $\mathcal{B}$. 


\begin{tabular}{|c|c|c|c|}
\hline Payload & $J_{1}, J_{2}\left[\mathrm{~kg} \mathrm{~m}^{2}\right]$ & $m, m_{3}[\mathrm{~kg}]$ & $d, r, l[\mathrm{~m}]$ \\
\hline Minimum & $0.591,0.628$ & $3.459,16.222$ & $0.120,0.295,0.480$ \\
\hline Maximum & $0.725,0.918$ & $3.459,23.222$ & $0.030,0.295,0.480$ \\
\hline \multicolumn{4}{|c}{ TABLE I }
\end{tabular}

GEOMETRIC AND INERTIAL PARAMETERS — FIRST DESIGN SOLUTION

\begin{tabular}{|c|c|c|c|c|c|c|}
\hline Manoeuvre & $\theta_{3}(0)\left[\left[^{\circ}\right]\right.$ & $P_{M}[\mathrm{~W}]$ & $n_{M}[\mathrm{rad} / \mathrm{s}]$ & $\tau_{r m s}[\mathrm{Nm}]$ & payload & slope \\
\hline R1 & $-90^{\circ}$ & 70.623 & 8.180 & 3.870 & $\max$ & $0 \%$ \\
\hline R2 & $90^{\circ}$ & 73.490 & 8.419 & 3.872 & $\max$ & $0 \%$ \\
\hline R3 & $90^{\circ}$ & 67.917 & 7.087 & 3.254 & $\min$ & $0 \%$ \\
\hline R4 & $-90^{\circ}$ & 67.917 & 6.780 & 3.264 & $\min$ & $0 \%$ \\
\hline R5 & $-90^{\circ}$ & 54.288 & 7.997 & 3.559 & $\min$ & $5 \%$ \\
\hline R6 & $-90^{\circ}$ & 54.228 & 8.184 & 4.036 & $\max$ & $5 \%$ \\
\hline R7 & $90^{\circ}$ & 54.672 & 7.997 & 3.670 & $\min$ & $5 \%$ \\
\hline R8 & $90^{\circ}$ & 62.469 & 8.281 & 4.038 & $\max$ & $5 \%$ \\
\hline R9 & $-90^{\circ}$ & 98.593 & 11.479 & 8.011 & $\min$ & $20 \%$ \\
\hline R10 & $-90^{\circ}$ & 112.157 & 11.760 & 7.865 & $\max$ & $20 \%$ \\
\hline R11 & $90^{\circ}$ & 100.679 & 11.513 & 7.987 & $\min$ & $20 \%$ \\
\hline R12 & $\mathbf{9 0}^{\circ}$ & $\mathbf{1 1 2 . 2 7 7}$ & $\mathbf{1 1 . 7 6 1}$ & $\mathbf{8 . 0 0 8}$ & $\max$ & $\mathbf{2 0} \%$ \\
\hline C1 & $90^{\circ}$ & 95.760 & 9.533 & 4.103 & $\max$ & $0 \%$ \\
\hline C2 & $-90^{\circ}$ & 95.301 & 9.530 & 4.148 & $\max$ & $0 \%$ \\
\hline C3 & $90^{\circ}$ & 94.639 & 9.632 & 4.682 & $\min$ & $0 \%$ \\
\hline C4 & $-90^{\circ}$ & 98.495 & 9.805 & 5.343 & $\min$ & $0 \%$ \\
\hline RT1 & $90^{\circ}$ & 28.052 & 2.797 & 3.262 & $\min$ & $0 \%$ \\
\hline RT2 & $-90^{\circ}$ & 27.106 & 2.738 & 2.135 & $\max$ & $0 \%$ \\
\hline \multicolumn{7}{|c|}{ TABLE II } \\
\hline
\end{tabular}

SIMULATED MANOEUVRES FOR ACTUATION SYSTEM DIMENSIONING

For the first design solution, the values of the geometric and inertial parameters of the robot, calculated for a minimum (i.e. no payload) and a maximum payload are displayed in Table I.

The robot direct dynamics and kinematics were simulated along with a multivariable state-feedback controller [3]; the desired profiles of the driving $(v)$ and steering $(\omega)$ velocities of the robot used in simulation were 40s-period square waves of $2 \mathrm{~m} / \mathrm{s}$ and $3.333 \mathrm{rad} / \mathrm{s}$ peak-to-peak amplitude, respectively. The simulated robot manoeuvres were intended to strongly excite the robot dynamics, see Table II. In the latter, $\theta_{3}(0)$ is the value of the tilt angle of the intermediate body at time zero, $P_{M}$ is the maximum power consumption observed in simulation, $n_{M}$ is the maximum absolute value of the angular velocity of the wheel, $\tau_{M}$ is the maximum absolute value of the torque applied to the wheel, $\tau_{r m s}$ is the root mean square torque applied to the wheel, R1...R4 refer to the rectilinear motion, $\mathrm{C} 1 \ldots \mathrm{C} 4$ refer to the circular motion, RT1. . RT4 refer to the rotation.

1) Planetary Gearhead Selection - Step One: R12 turns out to be the most interesting manoeuvre, whose observed values will be used as reference in the actuation system dimensioning. In this light, one needs a speed reducer with a maximum continuous torque of at least $\tau_{r m s}=8.008 \mathrm{Nm}$ and an intermittent torque of at least $\tau_{\max }=10 \mathrm{Nm}$. These requirements are fulfilled by a PG with $42 \mathrm{~mm}$ of chassis diameter (ceramic version) by Maxon [11]. Knowing the maximum recommended input speed of 8000rpm [11] of the foregoing PG and knowing the operating speed of the wheel

$$
n_{o p}=\frac{v}{r}=\frac{2 \mathrm{~m} / \mathrm{s}}{0.295 \mathrm{~m}}=6.780 \mathrm{rad} / \mathrm{s}=64.741 \mathrm{rpm},
$$

\begin{tabular}{|c|c|c|}
\hline Motor series & $\tau_{\text {cont }}[\mathrm{Nm}]$ & $\tau_{\text {stall }}[\mathrm{Nm}]$ \\
\hline Maxon RE 40, 150 W & 0.201 & 2.50 \\
\hline Maxon RE 35, 90W & 0.113 & 1.07 \\
\hline \multicolumn{2}{|c|}{ TABLE III }
\end{tabular}

TABLE III

MOTOR SELECTION - STEP ONE

one can easily compute the maximum reduction ratio $\varepsilon_{\max }=$ $8000 / n_{o p}=123.569$. One selects the three-stage PG that features the next smallest reduction ratio of $\varepsilon=338 / 3$ having a maximum efficiency of $\eta=72 \%$.

2) Motor Selection - Step One: Let us calculate speed and torque at the motor shaft: $n_{o p, m}=\varepsilon n_{o p}=7294.15 \mathrm{rpm}$, $\tau_{r m s, m}=1000 \tau_{r m s} /(\varepsilon \eta)=0.099 \mathrm{Nm}$ and $\tau_{\max , m}=$ $1000 \tau_{\max } /(\varepsilon \eta)=0.124 \mathrm{Nm}$. The $\tau_{r m s, m}$ value must be smaller than the motor continuous torque $\tau_{\text {cont }}$, in order to prevent the motor from overheating. Moreover, the stall torque $\tau_{\text {stall }}$ of the selected motor should usually exceed the required peak torque, i.e. $\tau_{\max , m}$. Furthermore, for the reasons outlined in a previous study [5], our motor choice is DC brushed; now, once we selected the PG Maxon GP42C $\emptyset 42 \mathrm{~mm}$ (203126) [11], we are bound to select one of the two DC brushed motors of Table III. The foregoing motors feature graphite brushes, which are better suited than precious metal ones for start/stop operations. Selection falls on the RE35 90W motor, whose $\tau_{\text {cont }}$ is high enough. Moreover, the motor should have a torque reserve so that it can function with somewhat unfavorable PG efficiency [11]. The additional torque requirement stemming from the $\tau_{\max , m}$ can be easily delivered by the motor, since the peak torque $(0.123 \mathrm{Nm})$ is not even twice as high as the continuous torque of the motor $(0.113 \mathrm{Nm})$.

After having selected the motor series one needs to select the motor winding. The RE $3590 \mathrm{~W}$ motor features an average speed-torque gradient of [11]

$$
\left.\frac{\Delta n}{\Delta \tau}\right|_{\text {av }}=\frac{(8.45+7.17)}{2}=0.00781 \mathrm{rpm} / \mathrm{Nm} .
$$

However, it should be noted that the winding of lowest resistance has a somewhat steeper gradient. The maximum speed observed in simulations is (see Table II) $n_{M}=112.309 \mathrm{rpm}$, which attains the following value when computed at the motor shaft: $n_{M, m}=\varepsilon n_{M}=12653.481 \mathrm{rpm}$. Hence, the desired no-load speed can be easily computed as [11]

$$
n_{0, \text { theor }}=n_{M, m}+\left.\frac{\Delta n}{\Delta \tau}\right|_{a v} \tau_{\max , m}=13620.584 \mathrm{rpm} .
$$

The extreme working point should be used in the calculation (maximum speed and torque), since the speed-torque line of the winding must run above all working points in the speed-torque diagram [11]. The no-load speed target must be achieved with the maximum voltage $U=33.6 \mathrm{~V}$ supplied by the power supply, the voltage drop over the power amplifier being negligible (see Subsection III.C); the latter defines the minimum speed-target constant

$$
K_{n, \text { theor }}=n_{0, \text { theor }} / U=405.374 \mathrm{rpm} / \mathrm{V} \text {. }
$$

Based on this calculation, motor 118776 [11] may be chosen, which corresponds to the winding with the next highest speed 


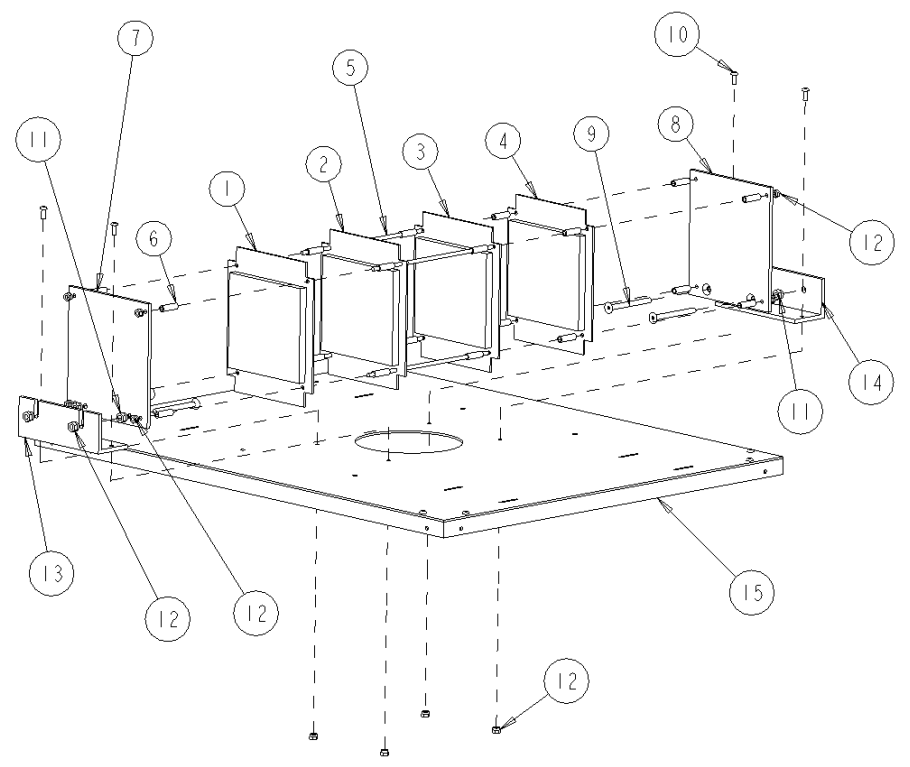

Fig. 5. Control unit exploded view - first design solution

constant $(491 \mathrm{rpm} / \mathrm{V})$ and has a second shaft end for mounting the encoder; however, the motor selection process stops because the 118776 motor has $\tau_{\text {cont }}=0.078 \mathrm{Nm}$, which is smaller than the RMS torque $\tau_{r m s, m}=0.099 \mathrm{Nm}$. Therefore, the selection process restarts from the motor series choice.

By selecting the RE40 150W series (see Table III) we have:

$$
\left.\frac{\Delta n}{\Delta \tau}\right|_{a v}=\frac{(4.11+3.04)}{2}=0.004 \mathrm{rpm} / \mathrm{Nm},
$$

while (1) and (2) become

$$
n_{0, \text { theor }}=13096.169 \mathrm{rpm} \quad K_{n, \text { theor }}=389.7678, \mathrm{rpm} / \mathrm{V} \text {. }
$$

We may select the motor of 148866 winding [11], which satisfies: $\tau_{\text {max }, m}<\tau_{\text {stall }}$. However, in order to avoid overheating of the motor windings and consequent permanent damages to the motor (the nominal voltage of this motor is half of $U$ ), the RE40 150W 148866 is discarded as well. Therefore, the actuation system dimensioning process needs to be restarted from the PG selection step.

3) Planetary Gearhead and Motor Selection - Step Two: The PG of the Maxon GP42C series with the next smallest reduction ratio is selected; it is a two-stage PG with the same efficiency as the previous one, but with $\varepsilon=74$. In this light we have: $n_{o p, m}=4758.463 \mathrm{rpm}, \tau_{r m s, m}=0.151 \mathrm{Nm}$ and $\tau_{\max , m}=0.190 \mathrm{Nm}$. From the latter, we are bound to select the RE40 series. As far as the motor winding is concerned we have: $n_{M, m}=8254.711 \mathrm{rpm}, n_{0, \text { theor }}=8933.299 \mathrm{rpm}$ and $K_{n, \text { theor }}=265.872 \mathrm{rpm} / \mathrm{V}$. We can then select the motor with winding 148867 , which has the next highest speed constant $(317 \mathrm{rpm} / \mathrm{V})$. Hence, The exact value of speed-torque gradient is [11]

$$
\frac{\Delta n}{\Delta \tau}=0.003 \mathrm{rpm} / \mathrm{Nm}
$$

while

$$
n_{0, \text { theor }}=n_{M, m}+\frac{\Delta n}{\Delta \tau} \tau_{\max , m}=8884.897 \mathrm{rpm}
$$

and

$$
K_{n, \text { theor }}=n_{0, \text { theor }} / U=264.431 \mathrm{rpm} / \mathrm{V} .
$$

The actual value of the speed constant $(317 \mathrm{rpm} / \mathrm{V})$ being higher than the latter, the motor runs faster than required which, however, can be compensated for by the controller. This selection ensures also that there is a speed regulating reserve of more than $15 \%$. Thus, even unfavorable manufacturing tolerances of the PG-motor systems are not a problem. It is easy to veirfy that the operating point of the robot is contained in the reccomended operating range of the motor at hand [11].

\section{Power Amplifier, Power Supply and Control Unit Dimen- sioning}

Knowing the torque constant of the selected motor, $K_{t}=$ $0.030 \mathrm{Nm} / \mathrm{A}$ [11], the desired maximum continuous current and the desired peak current are $i_{\text {cont }}=\tau_{r m s, m} / K_{t}=$ $5.011 \mathrm{~A}$ and $i_{\text {peak }}=\tau_{\max , m} / K_{t}=6.285 \mathrm{~A}$, respectively. The latter suggest the selection of the 25A8 series of PWM servo amplifiers for brushed motors by Advanced Motion Controllers [12], which features a maximum (internally limited) continuous current of $12 \mathrm{~A}$ and a peak current (lasting $2 \mathrm{~s}$ maximum and internally limited) of $25 \mathrm{~A}$.

In motion control applications, two important factors have to be taken into account when dimensioning the power supply: the internal resistance and the nominal voltage. Quasimoro is powered by four Nickel-metal Hydride battery packs arranged in series; each pack has seven Sanyo Twicell HR-D cells. This set-up is characterized by $V_{\text {nom }}=33.6 \mathrm{~V}$ and $R_{\text {in }}=$ $0.084 \Omega$ [13], which is low enough not to hamper the robot controller performance [3]. Moreover, the nominal voltage was selected as 1.4 times the nominal voltage of the motor for a better exploitation of the actuator.

The control architecture was dimensioned so as to resemble that of a successful robotic experimental test-bed developed at the McGill Centre for Intelligent Machines: SCOUT II [14], [15]. The control system will comprise a PC/104 stack of four boards (see Fig. 5), namely i) the computer board Lippert Cool Road Runner II; ii) the PC/104 power supply RTD EPWR104HR-25/25W; iii) the PC/104 quadrature decoder Microcomputer Systems MSI-P400 (2-CH); and iv) the PC/104 I/O board Micro/Sys MPC550 24DIO, 3TIMER, 16A/D, 4CH. D/A.

The command signals are sent by the user from a remote radio-frequency transmitter, a Tower Hobbies 4-channel 4THFM, which resembles the joystick of a an electrically propelled wheelchair; the command signals are then fed to the control unit by means of a radio frequency receiver, a Tower Hobbies System 3000 7-channel FM receiver, and the PC/104 I/O card.

\section{Robot Drive System and Chassis}

In order to align the wheel axes a cage made of four customfabricated aluminum alloy braces was designed, as shown 


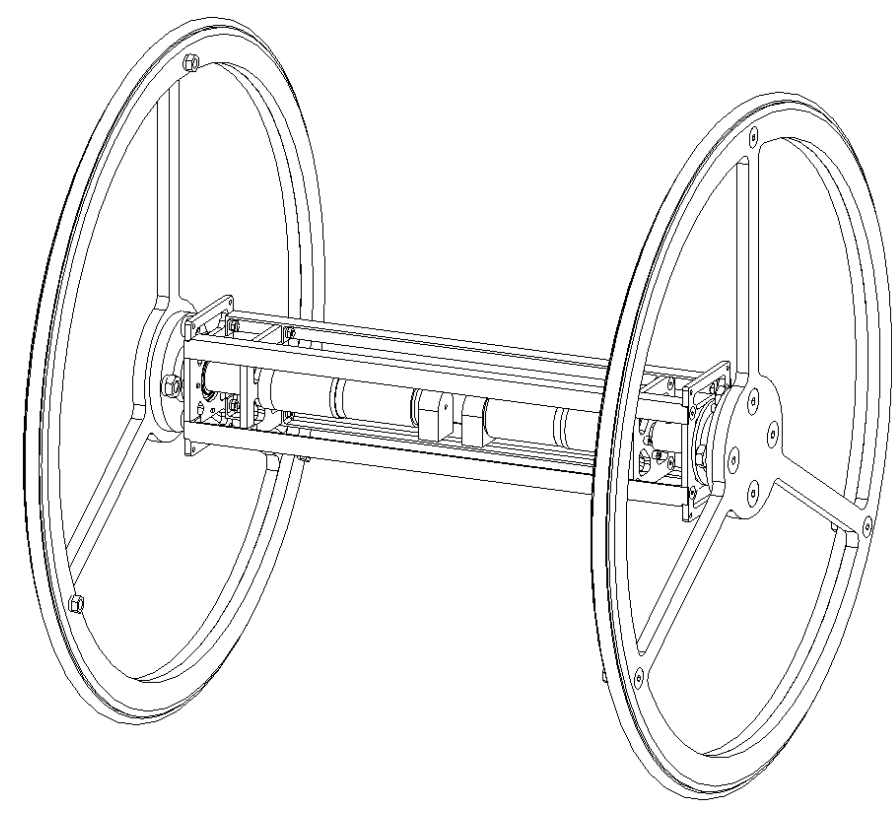

Fig. 6. Robot drive system - first design solution

in Fig. 6. This solution would guarantee the parallelism of the wheels with a corresponding benefit to the positioning accuracy of the robot.

\section{Second Design Solution}

In order to lower the overall mass centre of the robot, to limit the width of the robot and to contain the bending moment of the planetary gearhead output shaft, a timing belt transmission was considered. Moreover, in order to reduce the manufacturing costs a commercial bicycle wheel was chosen, as opposed to its custom-made counterpart.

These design corrections affected mainly the robot drive system. However, before discussing how the latter was redesigned we shall dwell on some design issues encountered when deriving this second solution.

1) Which commercial wheel?: An intense brain-storming session led us to select a commercial bicycle wheel. However, several wheel design solutions were considered before making the final decision. The main specifications for the wheel design were: minimum diameter of $590 \mathrm{~mm}$ and contact wheelground area as small as possible.

Given the application of the robot, we attempted to use the $24^{\prime \prime}$ Economy Wheel, with a standard round profile urethane snap-on tire, by Skyway, which represents a low cost alternative to the commercial $24^{\prime \prime}$ wheelchair alternative designed for the home health-care market [16]. Unfortunately, Skyway provides a metal keyway hub for wheels not larger than $20^{\prime \prime}$; the $24^{\prime \prime}$ wheels have a metal hub which is not suitable for hosting a keyway. Therefore, although the foregoing composite wheel by Skyway respects our specs, the interfacing with a custom-made hub would be rather complicated and expensive, as opposed to the solution outlined in Section V.

2) Wheel hub motors?: In order to fully exploit the commercial bicycle wheel solution, the conceptual design of a drive system featuring the Heinzmann hub-motor [17], one of the most successful actuators for electrical bicycles, was attempted. Using these motors would have allowed us to drastically reduce the overall dimensions of the robot actuation system and, correspondingly, to better exploit the interior part of the robot chassis. However, we discarded the Heinzmann hub-motor solution because $i$ ) it is at least twice as expensive as the Maxon motor solution of Subsubsection III.B, ii) no encoder can be readily custom-fitted to sense the position of the wheel shaft, and $i i i$ ) the integral gearbox, made of two mating spur gears, is not recommended for robotic applications.

3) Why timing belts?: In order to prevent the PG from failing upon impact between the wheel and obstacles, a transmission mechanism that would decouple the driving torque from the bending moment of the PG output shaft was designed. Although chains are generally, under the same transmission conditions, much cheaper than timing belts, a chain transmission was discarded for various reasons [18]: i) a chain is more suitable to long-term continuous running and power transmission with limited torque fluctuation; and $i$ ) produces undesirable speed variations stemming from the chordal action phenomenon.

Although suitable for applications like ours, which require precise positioning accuracy and repeatability, metal timing steel belts were discarded for the design complications introduced by the belt camber phenomenon [19]. Belt camber is the deviation of a belt edge from a straight line; for our application the estimated camber would be approximately $2 \mathrm{~mm}$. In order to cope with the latter one should resort to independently steerable pulleys [19], which will represent a sophistication that goes against the KISS approach.

A timing belt transmission system was selected because [20]:

- backlash between pulley and belt teeth is negligible;

- timing belts do not require lubrication; and

- timing belts do not slip and there is no relative motion between two elements in mesh.

However, the robot controller will have to account for mechanical-resonance related issues, once a timing belt is chosen. Mechanical resonance is a pervasive problem in servo systems [21], generally caused by the compliance of power transmission components. This compliance often reduces stability margins, forcing servo gains down and reducing machine performance, while mechanical instability can be achieved when a high-gain control law is applied to a compliantlycoupled motor and load [21].

4) Drive System Design: The second design solution is characterized by a bicycle wheel tightened to a custom-made shaft by means of a frictional connector; a timing belt takes care of the mechanical power transmission from the output shaft of the planetary gear to the custom-made shaft, as depicted ${ }^{2}$ in Fig. 7. Such a solution allows for redundant

\footnotetext{
${ }^{2}$ The timing belt was purposefully omitted in order to better show the transmission mechanism.
} 


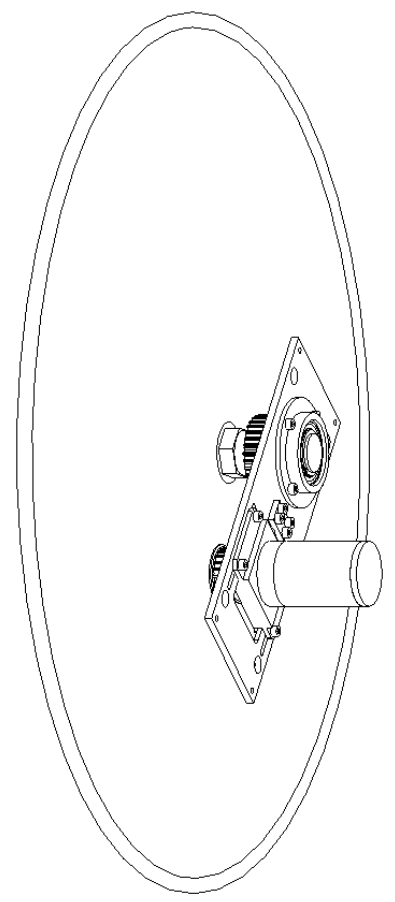

Fig. 7. Robot drive system — second design solution

sensing, since motion sensors can be readily assembled on the custom-made shaft 2 of Fig. 8 .

An exploded view of the drive system is reported in Fig. 8. With 1 we indicate the two frictional shaft locks which fasten the bicycle wheel 6 to shaft 2 . Note that we took away the bicycle wheel hub and bearings, we are only using the wheel rim and tire. With 4 we indicate a rolling bearing that allows shaft 2 to rotate with respect to the robot chassis, on which plate 10 is to be mounted. The actuation system, of which we report only the PG 5 , is housed in motor mount 9 , which is itself fastened to plate 10 . The motion is transmitted from the driving pulley 8 - fastened to PG 5 by means of a key and two set screws, not displayed here - to the driven pulley, which is mounted on shaft 2 by means of a key (two spacers will prevent the pulley from moving axially); device 3 is intended to retighten the timing belt, not displayed here.

This design solution turns out to be more cost-effective than the first onep; in fact, the machining costs are dramatically cut down by the selection of a commercial wheel. Moreover, the selection of a cheaper aluminum alloy, namely, Al 6061 (as opposed to Al 7075 featured by the first solution), for most of the components, helped to further reduce the overall robot cost.

\section{THIRD DESIGN SOlution}

Another design solution, the final one, was preferred to the previous ones. Instead of the custom-made shaft solution, which is rather expensive, a simpler and more cost-effective one was adopted, which uses the bicycle wheel shaft and bearings.

In Figs. 9 and 10 we show the robot under no-load and under full-load, respectively. The maximum payload consists of a 21

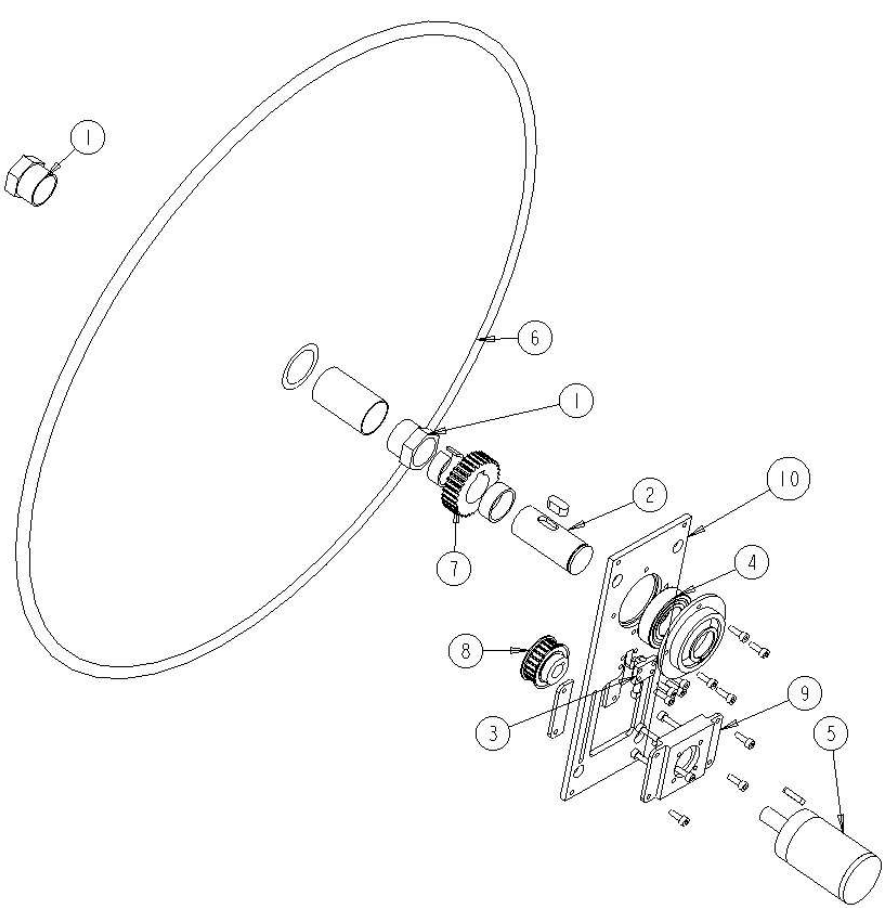

Fig. 8. Robot drive system exploded view - second design solution

soft-drink bottle, two food boxes (displayed in green) and two telephone books (displayed in blue). We can readily see how this design solution provides an optimum space utilization of the robot chassis volume.

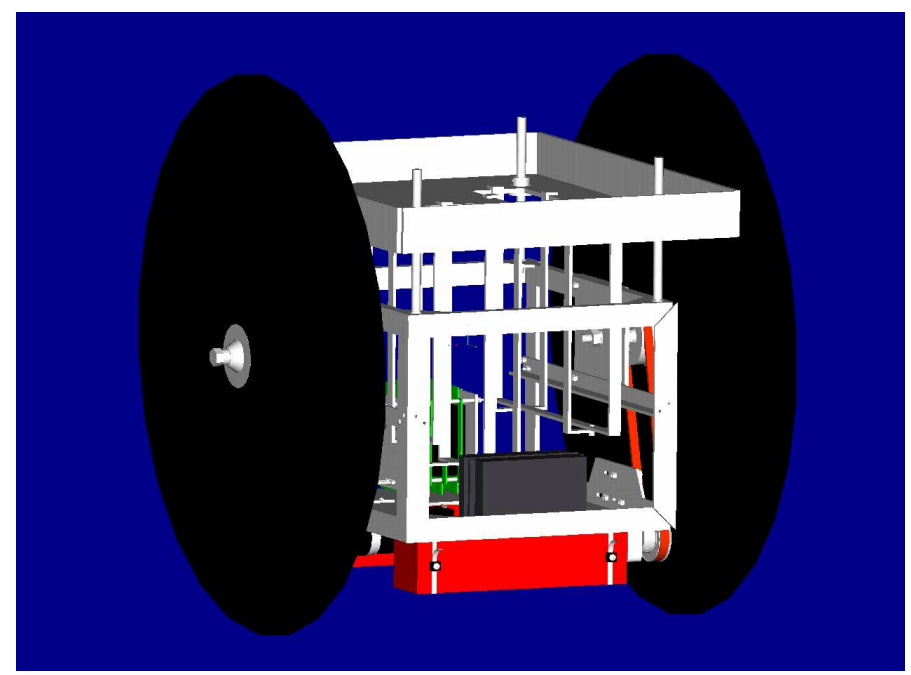

Fig. 9. Robot under no-load condition — third design solution

The layout of the electronic equipment was redesigned in such a way that it could be easily serviced, much like a drawer, as shown in Fig. 11.

The timing belt design features a $232.45 \mathrm{~mm}$ nominal centre distance at installation; considering a no more than $0.25^{\prime \prime}$ variation from assembly and manufacturing tolerances, the minimum centre distance is $226 \mathrm{~mm}$, as depicted in Fig. 12; in order to take into account the belt take-up phenomenon [20], which reflects in a maximum adjustment in the center distance 


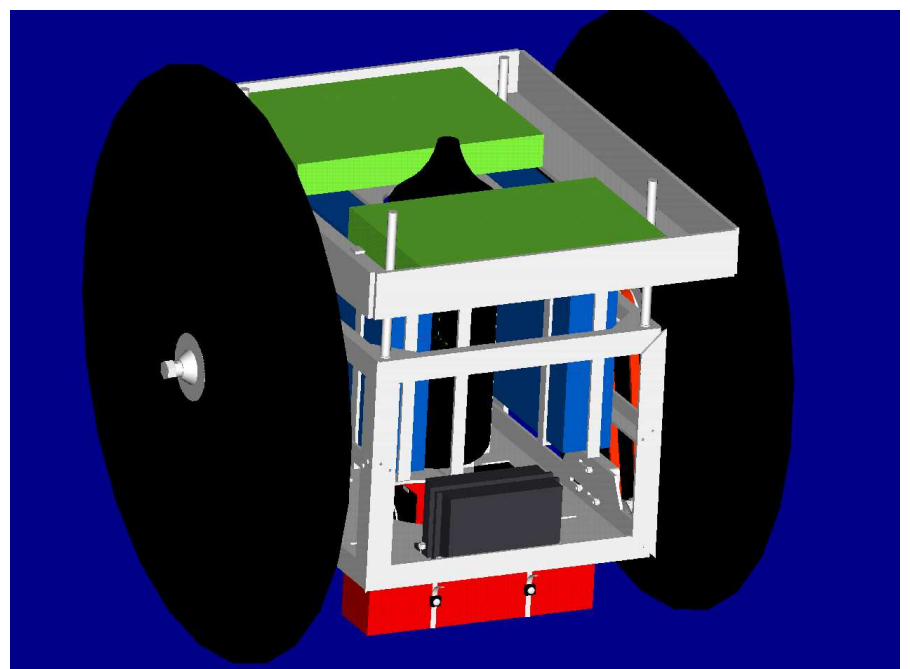

Fig. 10. Robot under full-load condition - third design solution
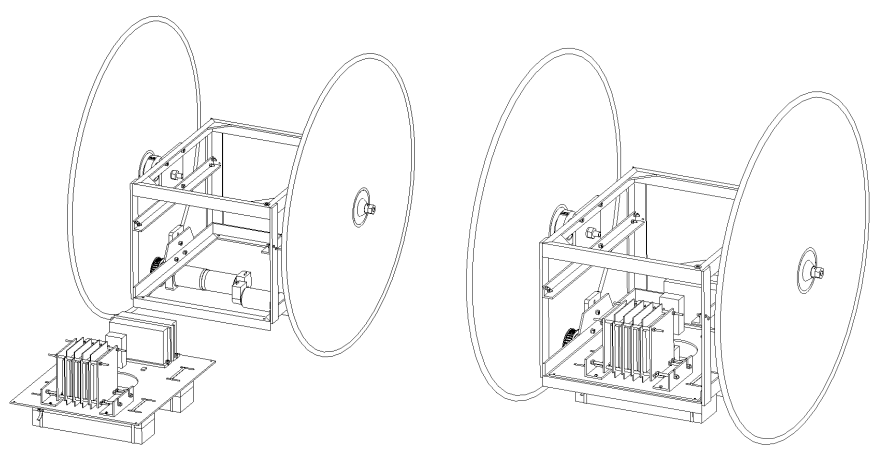

Fig. 11. Electronic equipment servicing - third design solution

of $0.5^{\prime \prime}$ from its nominal value, three slots were vertically machined on the motor mount. The reduction ratio achieved by the timing belt transmission is practically unity (1.18), the selected pulleys and belt being those of Table IV [20]. The pulleys are made of aluminum alloy and the timing belt is of the PowerGrip GT type made of neoprene with fibreglass tension members [20].

The payload holder, shown in Fig. 13, consists of a tray with three sub-frames, which are meant for holding books and bottles.

The robot chassis consists of 14 extruded aluminum alloy stock components, which are welded together, and two plates, namely the wheel plate and the motor mount that are screwed on the welded frame, as depicted in Fig. 14.

\section{Robot Control - Simulation Results}

\section{A. Controller Specifications}

The robot control system main tasks are $i$ ) positioning and orienting the payload, supported by the IB, on a flat surface and $i$ i) suppressing the oscillations of the IB, i.e. $\theta_{3, \infty}=$

\begin{tabular}{|c|c|c|}
\hline Driving pulley & Driven pulley & timing belt \\
\hline A6A55M034DF0912 & A6A54M040GT20 & A6R55M120090 \\
\hline
\end{tabular}

TIMING BELT TRANSMISSION COMPONENTS — THIRD DESIGN SOLUTION

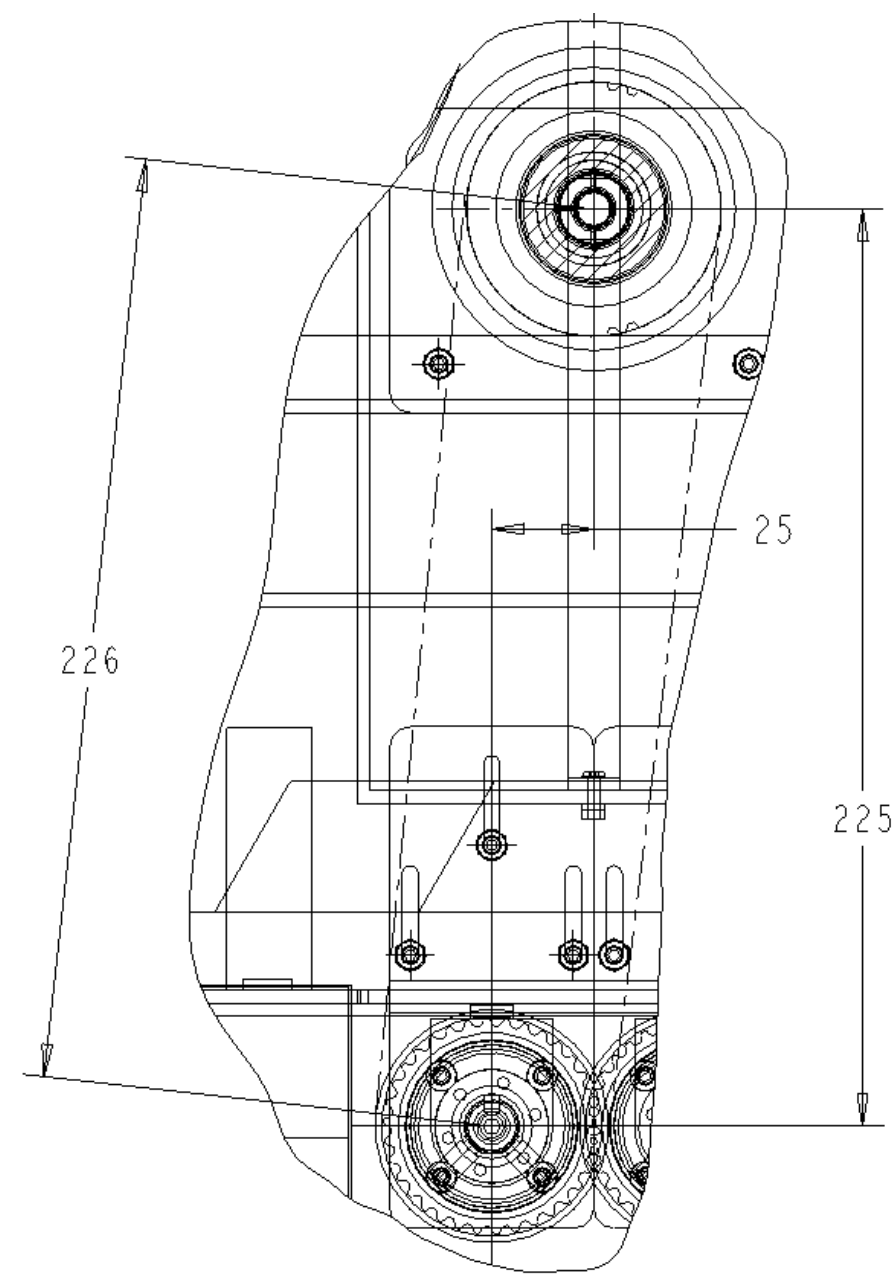

Fig. 12. Drive system section view - third design solution

\begin{tabular}{|l|l|}
\hline Rise time & $t_{r} \leq 3 \mathrm{~s}$ \\
\hline Overshoot & $M_{p} \leq 15 \%$ \\
\hline Settling Time & $t_{s, 1 \%} \leq 10 \mathrm{~s}$ \\
\hline
\end{tabular}

TABLE V

REQUIREMENTS ON THE TIME-RESPONSE $\mathbf{y}(t)$ OF THE SYSTEM

$\lim _{t \rightarrow \infty} \theta_{3}(t)=0$. Another functional requirement refers to the overshoot and $1 \%$ settling time of the tilt angle, namely, $M_{p, \theta_{3}} \leq 1.0467 \mathrm{rad}$ and $t_{s, 1 \%, \theta_{3}} \leq 10 \mathrm{~s}$, respectively. In terms of static performance the controller has to guarantee that $\mathbf{y}_{\infty}=\lim _{t \rightarrow \infty} \mathbf{y}(t)=\mathbf{y}_{d}=\left[\begin{array}{ll}\omega_{d} & v_{d}\end{array}\right]^{T}$, where $\mathbf{y}_{d}$ is the desired output vector, a.k.a. reference input vector, while $v_{d}$ and $\omega_{d}$ are the desired values of $v$ and $\omega$. The requirements on the time-response $\mathbf{y}(t)$ of the system (dynamic performance) are summarized in Table V.

\section{B. Simulation Results}

In order to validate the final design solution, simulation runs were conducted using Simulink ${ }^{\mathrm{TM}}$, implementing also the power supply, power amplifier and actuator models, and taking into account the reaction torques acting on the intermediate body. 


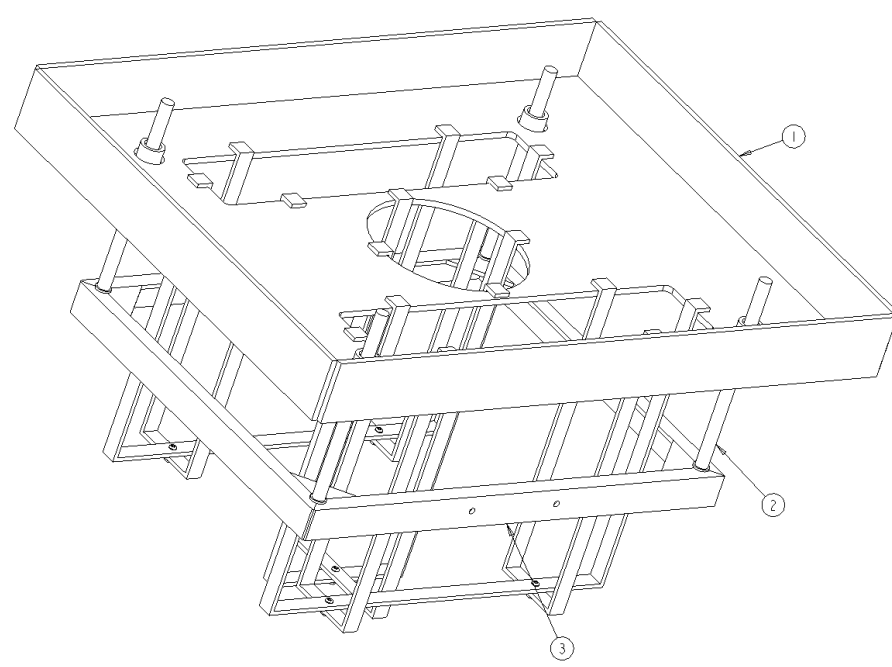

Fig. 13. Payload holder - third design solution

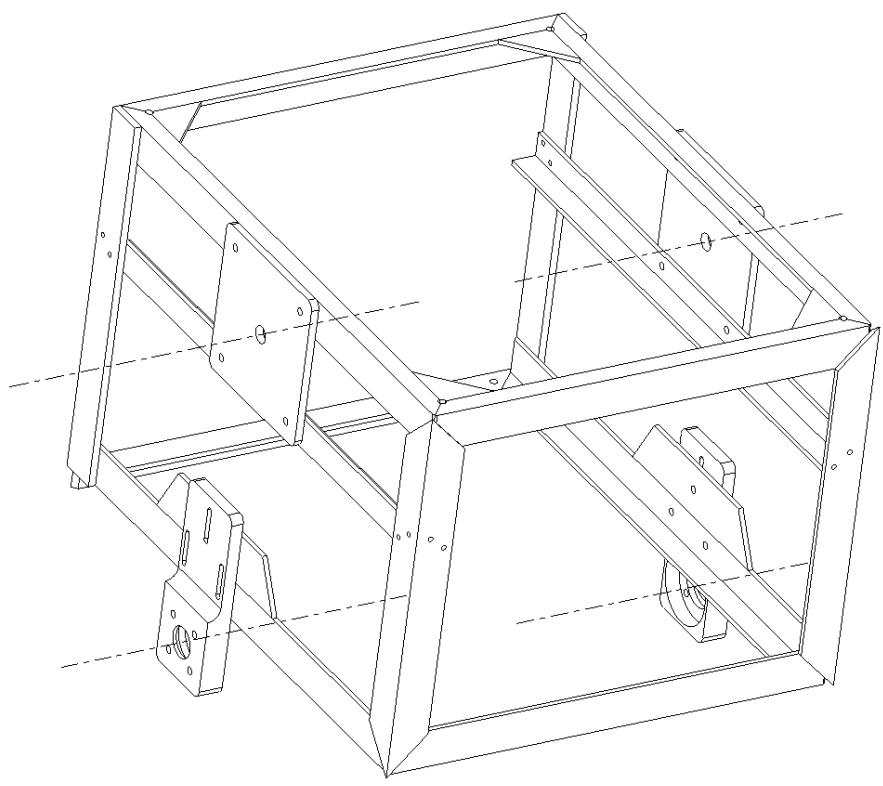

Fig. 14. Robot chassis - third design solution

The manoeuvres implemented in simulation are summarized in Table VI. With reference to $v_{d}\left(\omega_{d}\right)$, the \pm sign is used to distinguish the forward (clockwise rotation) from the backward rectilinear motion of the robot (counterclockwise robot rotation in place). The multivariable state feedback controller was designed according to a linear-quadratic regulator technique [3]; the controllers features the gain matrix $\mathbf{M}_{r}$ for reference input tracking and state feedback matrix $\mathbf{K}_{r}$, namely,

$$
\begin{gathered}
\mathbf{K}_{r}=\left[\begin{array}{cccc}
-1.8436 & 0.9129 & 0.0000 & -3.7032 \\
-1.8436 & 0.0000 & 0.9129 & -3.7032
\end{array}\right] \\
\mathbf{M}_{r}=\left[\begin{array}{cc}
3.0945 & -0.7422 \\
3.0945 & 0.7422
\end{array}\right] .
\end{gathered}
$$

Simulation results are represented by the plots of $\theta_{3}(t), v(t)$, and $\omega(t)$, as per Figs. 15-17. From the latter we can infer that the robot performance does not deteriorate upon changing the

\begin{tabular}{|c|c|c|}
\hline Maneuver Type & $v_{d}[\mathrm{~m} / \mathrm{s}]$ & $\omega_{d}[\mathrm{rad} / \mathrm{s}]$ \\
\hline Rectilinear Motion & \pm 2.000 & 0 \\
\hline Pure Rotation & 0 & \pm 2.500 \\
\hline Circular Motion & \pm 2.000 & \pm 2.500 \\
\hline \multicolumn{2}{|c}{ TABLE VI }
\end{tabular}

TYPE OF MANOEUVRES AND REFERENCE INPUTS

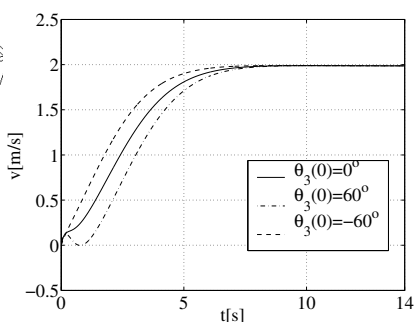

(a)

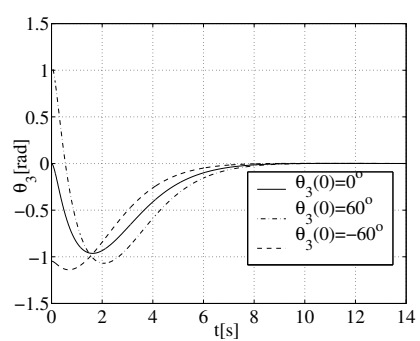

(b)
Fig. 15. Rectilinear motion: (a) $v$; (b) $\theta_{3}$.

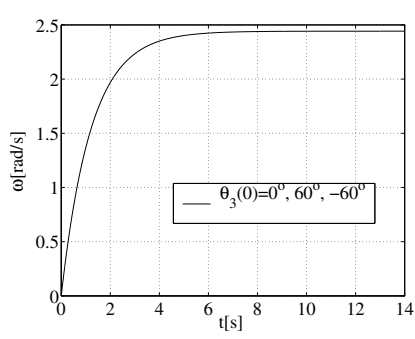

(a)

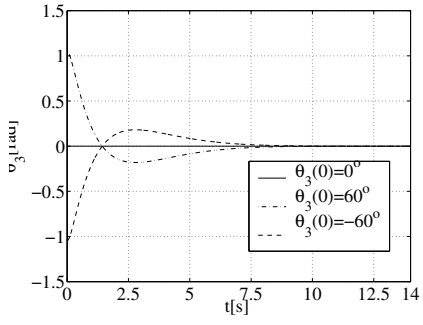

(b)
Fig. 16. Rotation: (a) $\omega$; (b) $\theta_{3}$.

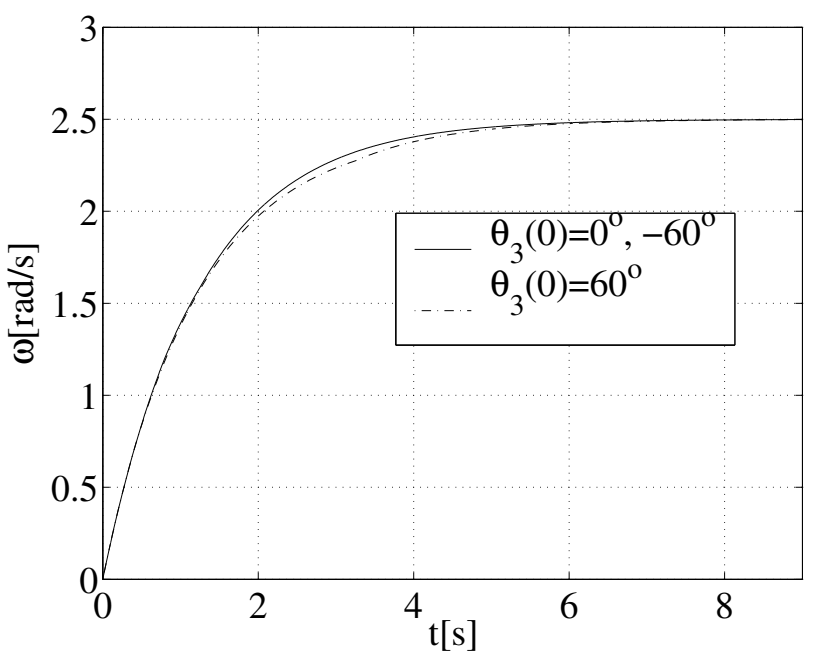

Fig. 17. Circular motion: $\omega$

initial configuration of the intermediate body with respect to the wheel axis $\left(\theta_{3}=\left\{0^{\circ}, 60^{\circ},-60^{\circ}\right\}\right)$, i.e. although purely linear, the controller at hand features in principle robustness with respect to changes of the robot initial configuration. The plots of $v(t)$ and $\theta_{3}(t)$ for the circular-motion case are identical to the ones of $v(t)$ and $\theta_{3}(t)$ for the rectilinear motion, as per plots of Fig. 15. Furthermore, the requirements on the static and dynamic performances of the robot are satisfied along with the specs on the tilt angle. Finally, from the plot of the motor torque, not reported here, neither the power 
amplifier nor the motor saturate under any of the foregoing manoeuvres.

\section{SUMmARY AND FUTURE WORK}

The mechanical design of a novel wheeled mobile robot was reported. Several design issues, such us wheel design, drive system design and controller dimensioning, were faced here. Three different design solution were laid out, while the control algorithm for the robot was successfully tested in simulation.

Future work entails: $i$ ) a standardization of a design methodology of wheel tires based on O-ring technology, ii) mechatronics design issues of the Quasimoro project, and iii) experimental validation.

\section{ACKNOWLEDGMENTS}

This work was made possible by NSERC, Canada's Natural Sciences and Engineering Research Council, under Research Grant A4532. The two-year "Hydro-Quebec" McGill Major Fellowship granted to Alessio Salerno is highly acknowledged.

\section{REFERENCES}

[1] R. Juvinall and K. Marshek, CRC Handbook of Chemistry and Physics, 2nd ed. John Wiley \& Sons, 1991.

[2] A. Salerno and J. Angeles, "A new family of two-wheeled mobile robots: Modeling and controllability," IEEE Trans. Robot., 2004, under review.

[3] — "The control of semi-autonomous two-wheeled robots undergoing large payload-variations," in Proc. IEEE Int. Conf. Robot. Automat., New Orleans, LA, 2004.

[4] T. Bräul, Embedded Robotics. Springer, 2003.

[5] A. Salerno and J. Angeles, "The preliminary design of a novel robot for human augmentation," in CCToMM Symposium on Mechanisms, Machines, and Mechatronics, Montreal, Canada, May 2003.

[6] Crossbow Technology, Inc., "Solid-state analog series tilt sensors cxta01, cxta02, datasheet." [Online]. Available at: http://www.xbow. com/Products/Product 'pdf files/Tilt pdf/6020-0014-01 'A \% CXTA.pdf

[7] Parker Seals, "Parker O-ring handbook," 2001, catalog ORD 5700A/US790.

[8] Fenner Drives, "Urethane o-ring — product specifications," 2004. [Online]. Available at: http://www.fennerindustrial.com/products/pdfs/ urethane tech.pdf

[9] Tampa Rubber \& Gasket Co., Inc., "Clear-go urethane o-rings." [Online]. Available at: http://www.tamparubber.com/

[10] H. Frederikse and D. Lide, Eds., CRC Handbook of Chemistry and Physics, 76th ed. CRC Press, 1996.

[11] Maxon Precision Motors 02/03 Catalogue, April 2002 ed., Maxon Motor.

[12] AMC Series 25A8 Servo Amplifiers Datasheet, Advanced Motion Controllers. [Online]. Available at: http://www.amc.com

[13] Twicell Sanyo Nichel-Metal Hydride Battery Datasheet, Sanyo Group. [Online]. Available at: http://www.sanyo.com/batteries/twicell.cfm

[14] I. Poulakakis, J. A. Smith, and M. Buehler, "Experimentally validated bounding moddels for the scout II quadrupedal robot," in Proc. IEEE Int. Conf. Robot. Automat., New Orleans, LA, 2004.

[15] J. A. Smith and I. Poulakakis, "Rotary gallop in the untethered quadrupedal robot Scout II," in IEEE/RSJ Int. Conf. Intell. Robots and Syst., Sendai, Japan, 2004, accepted for publication.

[16] Skyway Machine, Inc., "Skyway wheelchair wheels." [Online]. Available at: http://www.skywaytuffwheels.com/

[17] Heinzmann GmbH, "Heinzmann hub-motors," in German. [Online]. Available at: http://www.estelle.de/motoren.asp

[18] M. Kanehira, T. Otani, M. Yoshikawa, and T. Takahashi, The Complete Guide to Chain, M. Kanehira and K. Otoshi, Eds. Tsubaki. [Online]. Available at: http://www.ustsubaki.com/

[19] Belt Technologies, Inc., "Metal timing belts." [Online]. Available at: http://www.belttechnologies.com

[20] Stock Drive Products/Sterling Instruments, Handbook of Timing Belts, Chains \& Friction Drives, 1991. [Online]. Available at: http://www.sdp-si.com/
[21] G. Ellis, "Cures for mechanical resonance in industrial servo systems," in Danaher Motion Training. [Online]. Available at: http://www2.danahermotion.com/service/training/articles/index.html 